\title{
Identification of candidate genes for the diagnosis and treatment of cholangiocarcinoma using a bioinformatics approach
}

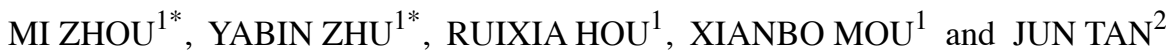 \\ ${ }^{1}$ Department of Cell Biology,The Medical School of Ningbo University, Ningbo, Zhejiang 315211; ${ }^{2}$ Department of \\ Hepatology, HwaMei Hospital, University of Chinese Academy of Sciences, Ningbo, Zhejiang 315010, P.R. China
}

Received January 10, 2019; Accepted August 20, 2019

DOI: $10.3892 / \mathrm{ol} .2019 .10904$

\begin{abstract}
Cholangiocarcinoma (CCA) is a biliary epithelial tumor with poor prognosis. As the key genes and signaling pathways underlying the disease have not been fully elucidated, the aim of the present study was to improve the understanding of the molecular mechanisms associated with CCA. The microarray datasets GSE26566 and GSE89749 were downloaded from the Gene Expression Omnibus and differentially expressed genes (DEGs) between CCA and normal bile duct samples were identified. Gene and pathway enrichment analyses were performed, and a protein-protein interaction network was constructed and analyzed. A total of 159 DEGs and 10 hub genes were identified. The functions and pathways of the DEGs were mainly enriched in 'heparin binding', 'serine-type endopeptidase activity', 'calcium ion binding', 'pancreatic secretion', 'fat digestion and absorption' and 'protein digestion and absorption'. Survival analysis revealed that the upregulated expression of carboxypeptidase B1 and Kruppel like factor 4 was significantly associated with lower overall survival rate. In summary, the present study identified DEGs and hub genes associated with CCA,
\end{abstract}

Correspondence to: Dr Jun Tan, Department of Hepatology, HwaMei Hospital, University of Chinese Academy of Sciences, 41 Xibei Road, Ningbo, Zhejiang 315010, P.R. China

E-mail: t-doctor@163.com

*Contributed equally

Abbreviations: CCA, cholangiocarcinoma; GEO, Gene Expression Omnibus; DEGs, differentially expressed genes; PPI, protein-protein interaction; EMT, epithelial-mesenchymal transition; GO, Gene Ontology; KEGG, Kyoto Encyclopedia of Genes and Genomes; DAVID, Database for Annotation, Visualization and Integrated Discovery; STRING, Search Tool for the Retrieval of Interacting Genes; MCODE, Molecular Complex Detection; BP, biological process; $\mathrm{CC}$, cell component; $\mathrm{MF}$, molecular function

Key words: cholangiocarcinoma, bioinformatics analysis, differentially expressed genes, Kaplan-Meier curves which may serve as potential diagnostic and therapeutic targets for the disease.

\section{Introduction}

Cholangiocarcinoma (CCA) is a diverse epithelial malignancy originating in the cholangiocytes, the epithelial cells of the bile duct. The incidence of CCA has increased globally over the past few decades (1). Chronic infection and inflammation due to liver fluke infection, sclerosing cholangitis and hepatitis $\mathrm{C}$ and $\mathrm{B}$ virus infections serve a key role in cholangiocarcinogenesis, possibly through the accumulation of genetic and epigenetic changes that result in abnormalities in oncogenes and tumor suppressor genes (2,3). The most commonly mutated genes in CCA, including KRAS proto-oncogene GTPase $(K R A S)$, tumor protein p53 (TP53), B-Raf proto-oncogene, serine/threonine kinase $(B R A F)$, BRCA1 associated protein 1(BAPl), and SMAD family member 4 (SMAD4), are associated with cell signaling pathways (for example, MAPKs signaling and TGF- $\beta$ signaling), cell cycle control and chromatin dynamics $(2,4)$. Previous studies have demonstrated that $K R A S$ point mutation in intrahepatic CCA (iCCA) may affect patient prognosis (5). Furthermore, mutations in $K R A S$ and TP53 in mature cholangiocytes and hepatocytes may cause iCCA (6). A combination of mitogen-activated protein kinase kinase $1 / 2$ and $B R A F$ inhibitors has been shown to be effective in patients with iCCA harboring the $B R A F$ V600E mutation (7). Activation of the transforming growth factor- $\beta / \operatorname{Smad} 4$ signaling pathway accelerates CCA cell invasion and migration via the epithelial-mesenchymal transition (EMT) (8). Knockdown of BAPl increases CCA cell proliferation, whereas overexpression of wild-type $B A P 1$ significantly inhibits cell proliferation, suggesting that $B A P I$ exhibits tumor suppressive effects (9). Despite significant efforts to elucidate the pathogenesis of CCA, the precise molecular mechanisms involved remain unclear. Therefore, the aim of the present study was to investigate the molecular mechanisms involved in the pathogenesis of CCA and to identify potential therapeutic targets.

In recent years, microarray technology has attracted attention due to its ability to rapidly and simultaneously quantify the expression levels of several genes, and is particularly suitable for the screening of differentially expressed genes (DEGs) (10). 
Several microarray-based studies on CCA have identified numerous DEGs $(11,12)$. However, previous reports were limited to independent microarray analysis or single cohort studies (13). Therefore, in order to identify more accurate and practical biomarkers, the present study analyzed two mRNA microarray datasets (GSE26566 and GSE89749), downloaded from the Gene Expression Omnibus (GEO), and screened for DEGs between cholangiocarcinoma and normal bile duct samples. Gene Ontology (GO) and Kyoto Encyclopedia of Genes and Genomes (KEGG) pathway enrichment analyses were subsequently performed and a protein-protein interaction (PPI) network was constructed. The results obtained in the present study may aid the early diagnosis and treatment of CCA.

\section{Materials and methods}

Microarray data. The current study aimed to elucidate the potential key candidate genes in CCA. Two gene expression profiles, GSE26566 (11) and GSE89749 (12), were downloaded from the GEO database (ncbi.nlm.nih.gov/geo). The GSE26566 dataset consisted of an mRNA expression profile of 104 CCA and 6 normal bile duct samples, while the GSE89749 dataset included 118 CCA and 2 normal bile duct samples. The normal bile duct samples were obtained from healthy controls. The samples in the GSE26566 dataset were obtained from three countries (Australia, Belgium and the United States of America) and the samples in GSE89749 dataset were obtained from ten countries (Singapore, Romania, Thailand, Italy, France, Korea, Brazil, Taiwan, China and Japan).

DEGs screening. The DEGs between CCA and normal bile duct samples were identified using GEO2R (www.ncbi.nlm. nih.gov/geo/geo2r), an interactive online tool used to identify DEGs by comparing samples from the GEO database. The cut-off criteria for the selection of DEGs were $\mathrm{P}<0.01$ and a $\mid \log$ fold-changel $\geq 1$. Default settings were used as the screening criteria throughout the entire bioinformatics analysis process, as has been reported in previous studies $(14,15)$.

$G O$ and KEGG analyses of DEGs. GO (geneontology.org) and KEGG (www.genome.jp/kegg) enrichment analyses of the DEGs were performed using the Database for Annotation, Visualization and Integrated Discovery (DAVID version 6.8; david.ncifcrf.gov) with $\mathrm{P}<0.05$ as the cut-off criterion. DAVID provides a comprehensive set of gene functional annotation information to extract biological information (16).

PPI network of DEGs. The PPI network was constructed using Cytoscape version 3.7.0 software (www.cytoscape.org) (17) based on the Search Tool for the Retrieval of Interacting Genes (STRING version 11.0; string-db.org). STRING is a system for searching for interactions between proven and predicted proteins (18). In the present study, genes with a combined score of $>0.4$ were considered significant. Sub-modules of the PPI network were analyzed using the Cytoscape plug-in Molecular Complex Detection (MCODE version 1.5.1) (19) with the criteria set as follows: MCODE score $>4$, node score cut-off $=0.2$, degree cut-off $=2$, max depth $=100$ and $k$-core $=2$. GO and KEGG analyses for the genes in the most significant sub-module were subsequently performed using Metascape version 3.5 (metascape.org) (20).

Hub gene selection and analysis. The top 10 genes ranked by network degree were selected as the hub genes. The corresponding proteins may be key candidate proteins with important physiological regulatory functions. GO and KEGG analyses for the hub genes were subsequently performed using Metascape version 3.5. A network of the genes and their co-expression genes was analyzed using cBioPortal (cbioportal.org), as described previously $(21,22)$. The University of California, Santa Cruz Cancer Genomics Browser (genome-cancer.ucsc. edu) was used for the hierarchical clustering of the hub genes, and for examining the association between changes in the hub genes and the Child-pugh classification grade or days to death (23). Survival analyses were performed to assess the prognostic value of the hub genes identified in the present study in CCA. Kaplan-Meier analysis in the cBioPortal online platform was used for overall survival rate and disease-free survival analyses of the hub genes based on a dataset from The Cancer Genome Atlas (TCGA; cancergenome.nih.gov/) which contained 51 CCA samples.

\section{Results}

DEGs screening. A total of 1,870 and 591 DEGs between CCA and normal bile duct samples were identified in the GSE26566 and GSE89749 datasets, respectively. The overlap between the two datasets included 159 DEGs (135 upregulated and 24 downregulated genes; Fig. 1).

$G O$ and KEGG enrichment analysis of DEGs. Candidate DEGs were subjected to function and pathway enrichment analysis in DAVID. GO analysis included biological processes (BP), cell components (CC) and molecular functions (MF). BP results suggested that the DEGs were mainly enriched in 'cell adhesion', 'digestion' and 'defense response to gram-positive bacteria' (Fig. 2A). CC results revealed that the DEGs were mainly enriched in 'extracellular space', 'proteinaceous extracellular matrixes' and 'extracellular regions' (Fig. 2B). MF results suggested that the DEGs were mainly enriched in 'heparin binding', 'calcium ion binding' and 'serine-type endopeptidase activity' (Fig. 2C). The KEGG pathway analysis suggested that the DEGs were mainly associated with 'pancreatic secretion', 'fat digestion and absorption' and 'protein digestion and absorption' (Fig. 2D).

PPI network of the DEGs. A PPI network containing 84 nodes and 155 edges was constructed using Cytoscape software based on the STRING database (Fig. 3A). The most significant sub-module was extracted from the PPI network complex using MCODE (Fig. 3B). The functions of the genes in the aforementioned sub-module were analyzed using the online tool Metascape. The sub-module consisted of 7 nodes and 17 edges, which were mainly associated with 'cell chemotaxis', 'G-protein coupled receptor signaling pathway' and 'positive regulation of response to external stimulus' (Fig. 3C).

Hub gene selection and analysis. The top 10 genes ranked by network degree were selected as hub genes and are presented in 
Table I. Functions of the 10 hub genes.

\begin{tabular}{|c|c|c|}
\hline Gene symbol & Full name & Function \\
\hline$I L-6$ & Interleukin 6 & $\begin{array}{l}\text { Encodes a cytokine that functions in inflammation and the maturation } \\
\text { of B cells }\end{array}$ \\
\hline$M Y C$ & MYC proto-oncogene & $\begin{array}{l}\text { Proto-oncogene that encodes a nuclear phosphoprotein that plays a role } \\
\text { in cell cycle progression, apoptosis and cellular transformation }\end{array}$ \\
\hline$S S T$ & Somatostatin & Inhibits the release of numerous secondary hormones \\
\hline CXCL12 & CXC motif chemokine ligand 12 & $\begin{array}{l}\text { Plays a role in a number of cellular functions, including embryogenesis, } \\
\text { immune surveillance, inflammation response, tissue homeostasis and } \\
\text { tumor growth and metastasis }\end{array}$ \\
\hline$N P Y$ & Neuropeptide Y & $\begin{array}{l}\text { Influences several physiological processes, including cortical } \\
\text { excitability, stress response, food intake, circadian rhythms and cardio- } \\
\text { vascular function }\end{array}$ \\
\hline LPARl & Lysophosphatidic acid receptor 1 & $\begin{array}{l}\text { Encodes the lysophosphatidic acid receptor, mediate diverse biologic } \\
\text { functions, including proliferation, platelet aggregation, smooth muscle } \\
\text { contraction, chemotaxis, and tumor cell invasion. }\end{array}$ \\
\hline$P L A 2 G 1 B$ & Phospholipase A2 group 1B & $\begin{array}{l}\text { Encodes a secreted member of the phospholipase A } 2 \text { class of enzymes. } \\
\text { The enzyme may be involved in several physiological processes } \\
\text { including cell contraction, cell proliferation and pathological response. }\end{array}$ \\
\hline$C P B 1$ & Carboxypeptidase B1 & $\begin{array}{l}\text { Carboxypeptidase B1 is a highly tissue-specific protein and is a useful } \\
\text { serum marker for acute pancreatitis and dysfunction of pancreatic } \\
\text { transplants. }\end{array}$ \\
\hline PNLIP & Pancreatic lipase & Encodes an enzyme involved in the digestion of dietary fats \\
\hline KLF4 & Kruppel like Factor 4 & $\begin{array}{l}\text { The encoded zinc finger protein is required for normal development of } \\
\text { the barrier function of skin. The encoded protein is thought to control } \\
\text { the G1-to-S transition of the cell cycle following DNA damage by } \\
\text { mediating the tumor suppressor gene p53. }\end{array}$ \\
\hline
\end{tabular}

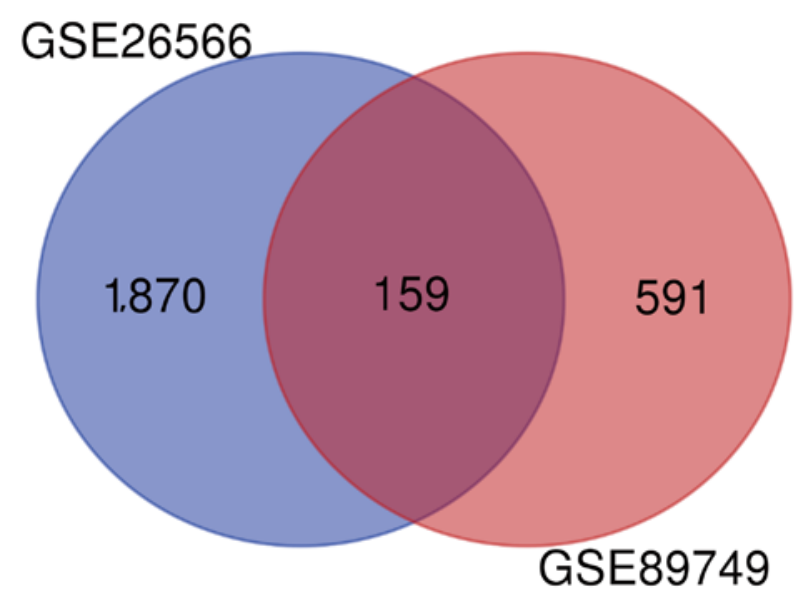

Figure 1. Venn diagram of the DEGs between cholangiocarcinoma and normal bile duct samples in the GSE26566 and GSE89749 datasets. A total of 159 overlapping DEGs were identified between the two datasets. DEGs, differentially expressed genes.

Table I. KEGG pathway analysis revealed that hub genes were mainly associated with the activation of the phosphoinositide 3-kinase-protein kinase B (Akt) signaling pathway (Table SI). A network of the hub genes and their co-expression genes was analyzed using cBioPortal (Fig. 4A). PLA2G1B and $P N L I P$ did not interact with other genes in the network, thus, only eight hub genes appeared in the network. Hierarchical clustering revealed that among the patients with CCA with upregulated hub genes, the patients were classified as grade $\mathrm{B}$ using the Child-pugh classification grade, and the days to death value was relatively small (Fig. 4B). The expression of $M Y C$ and LPARI did not an alteration in the TCGA dataset. As a result, the survival curves of 8 out of the 10 hub genes are presented in the current study. The 6 hub genes that did not 
A

Cell adhesion Digestion

Defense response to Gram-positive bacterium Defense response to Gram-negative bacterium

Proteolysis

Extracellular matrix assembly Regulation of blood pressure Bleb assembly Nervous system development Response to yeast Lipid digestion Lipid catabolic process Positive regulation of gene expression Negative regulation of transcription from RNA polymerase II promoter Positive regulation of transcription from RNA polymerase II promoter Positive regulation of fibroblast proliferation Platelet degranulation Positive regulation of smooth muscle cell proliferation Negative regulation of catecholamine secretion Negative regulation of apoptotic process s.

B Extracellular spac Proteinaceous extracellular matrix Extracellular region Extracellular matrix Platelet alpha granule membrane Integral component of plasma membrane

External side of plasma membrane

Plasma membrane Extracellular exosome Membrane raft Elastic fiber

Sarcolemma Platelet dense granule membrane Collagen trimer Cell surface -

2.5

C

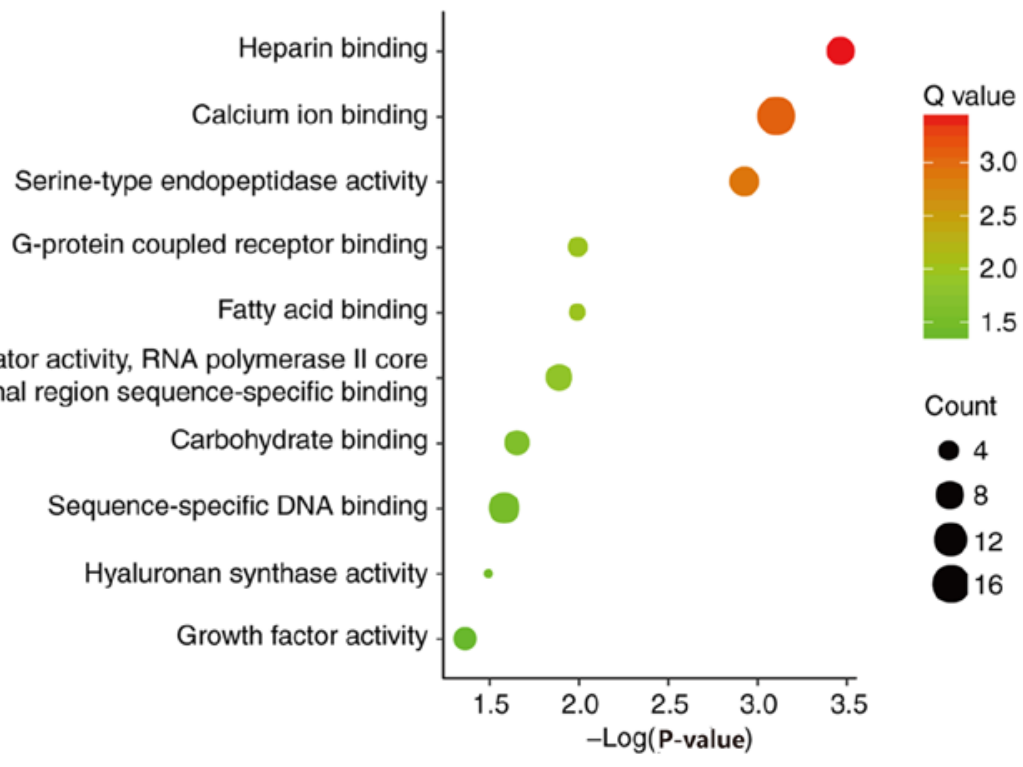

Figure 2. Gene ontology analysis, including (A) biological process, (B) cell component and (C) molecular function. 


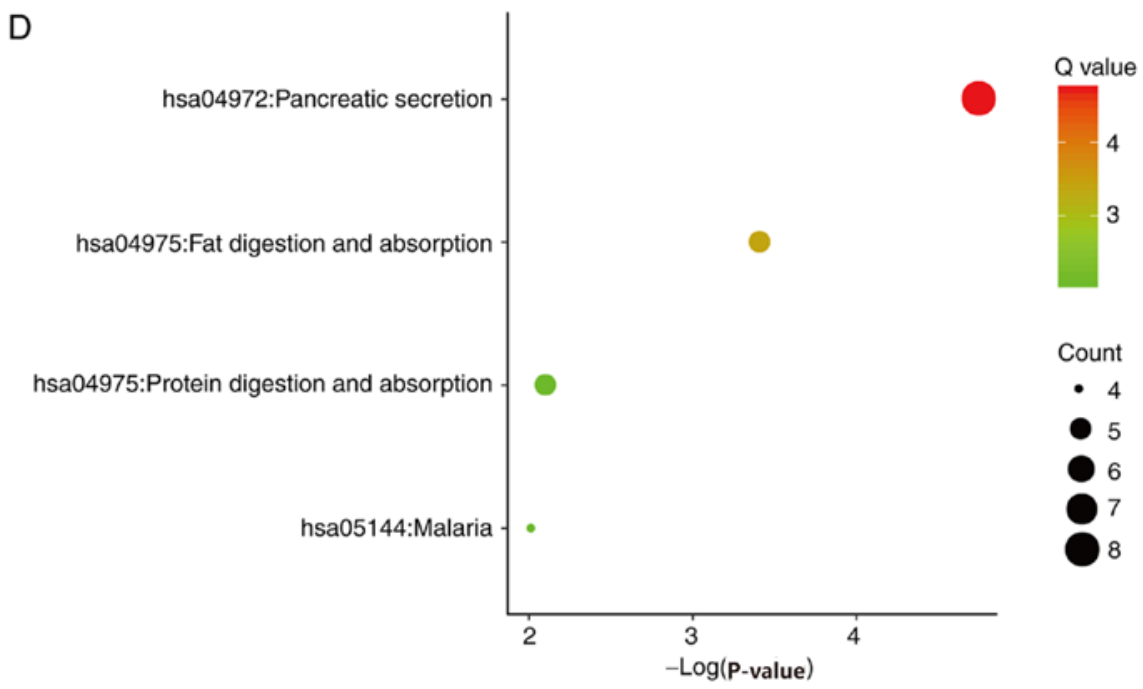

Figure 2. Continued. (D) Kyoto Encyclopedia of Genes and Genomes analysis of the differentially expressed genes.

A

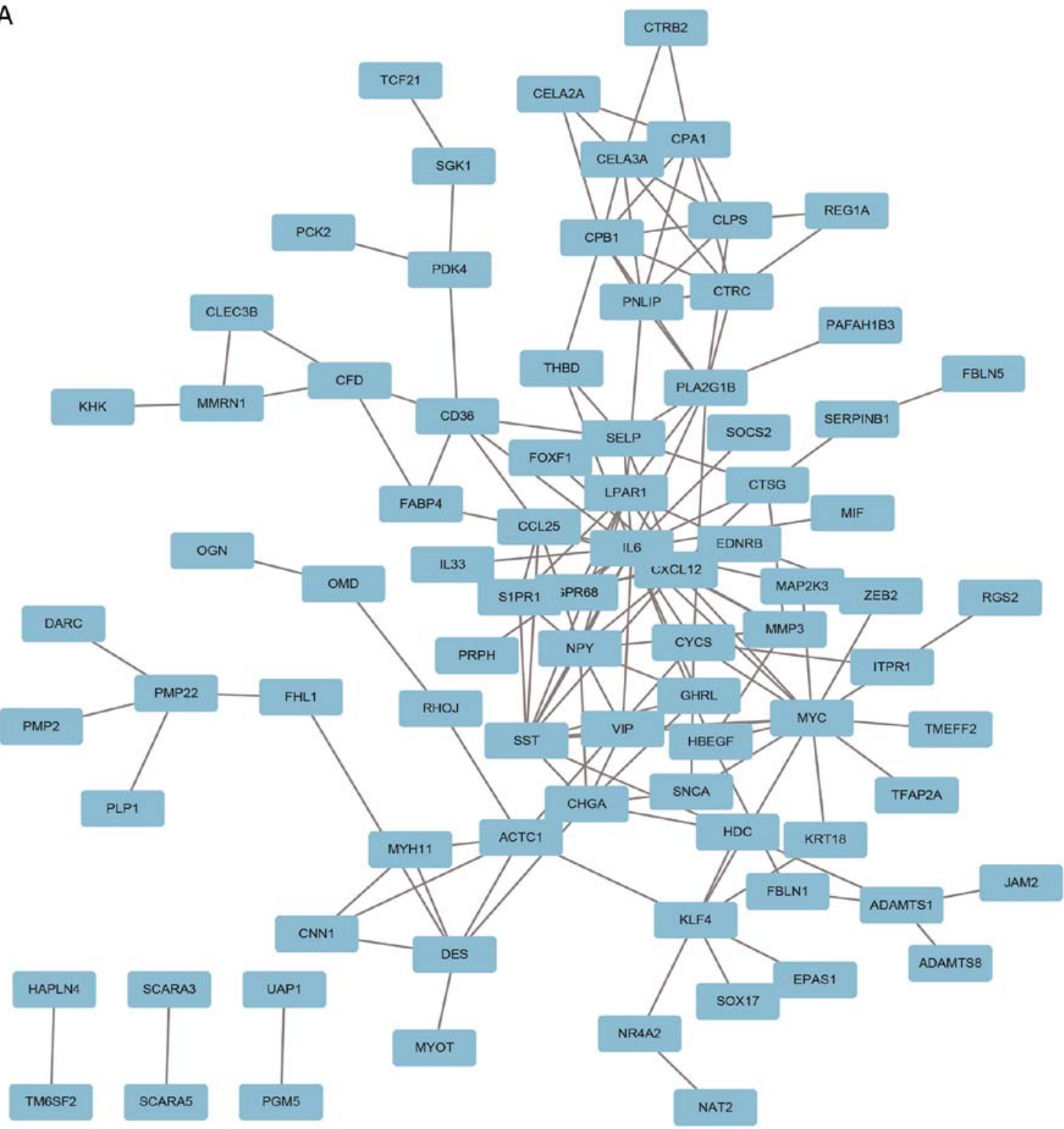

Figure 3. PPI network and the most significant sub-module of DEGs. (A) The PPI network was constructed using the Search Tool for the Retrieval of Interacting Genes and Cytoscape software. 

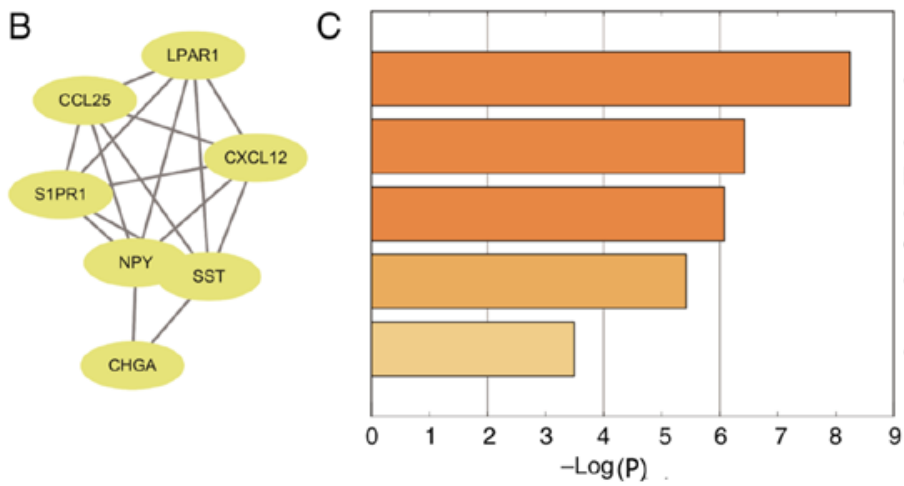

GO:0060326: Cell chemotaxis

GO:0007187: G-protein coupled receptor signaling pathway, coupled to cyclic nucleotide second messenger GO:0032103: Positive regulation of response to external stimulus

GO:0005179: Hormone activity

GO:0008015: Blood circulation

Figure 3. Continued. (B) The most significant sub-module consisted of seven nodes and 17 edges. (C) GO and Kyoto Encyclopedia of Genes and Genomes pathway enrichment analysis of the DEGs in the most significant sub-module. PPI, protein-protein interaction; DEGs, differentially expressed genes; GO, Gene Ontology.

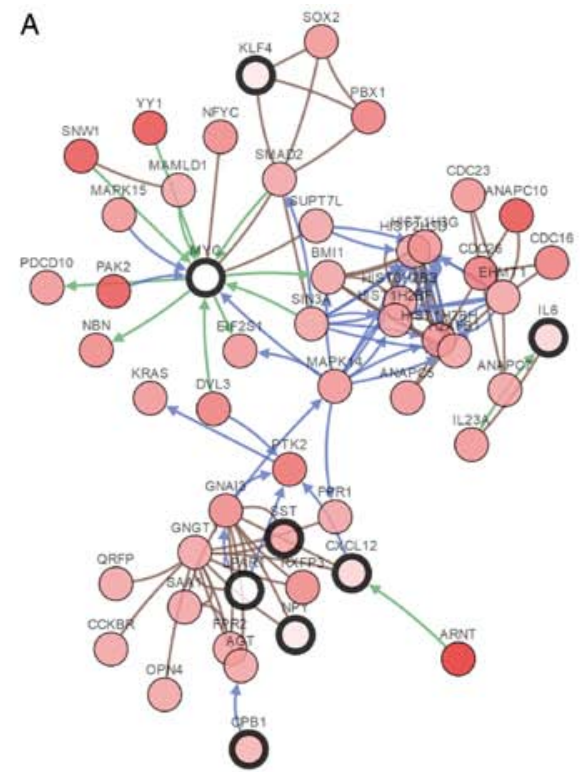

B

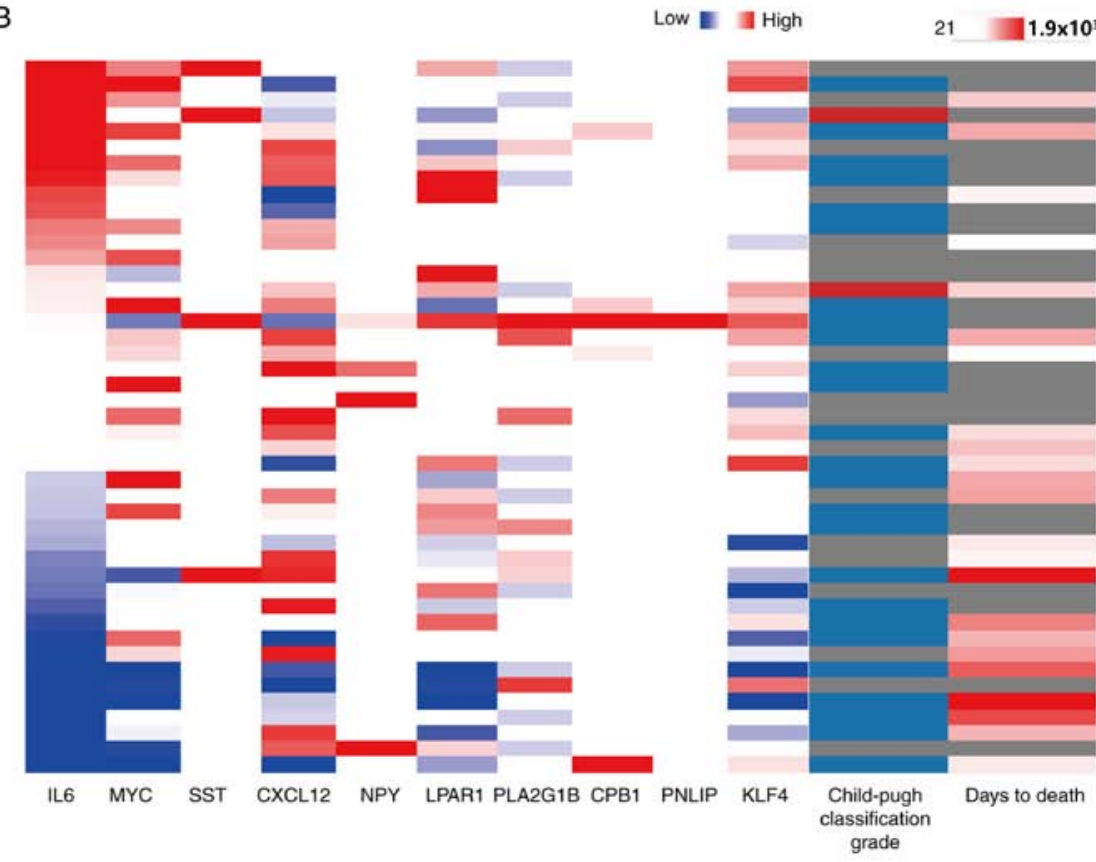

Figure 4. (A) Hub genes and their co-expression genes were analyzed using cBioPortal. Nodes with a bold black outline represent hub genes. Nodes with a thin black outline represent the co-expression genes. (B) Hierarchical clustering of the hub genes was performed using the University of California, Santa Cruz Cancer Genomics Browser. The genomic heat map is presented on the left and the clinical heat map is presented on the right. Grey represents no data. Red and blue represent upregulation and downregulation, respectively. The blue and red cells in the Child-Pugh classification column represents grade A and B, respectively. 
Cases with alteration(s) in query gene(s)

- Cases without alteration(s) in query gene(s)
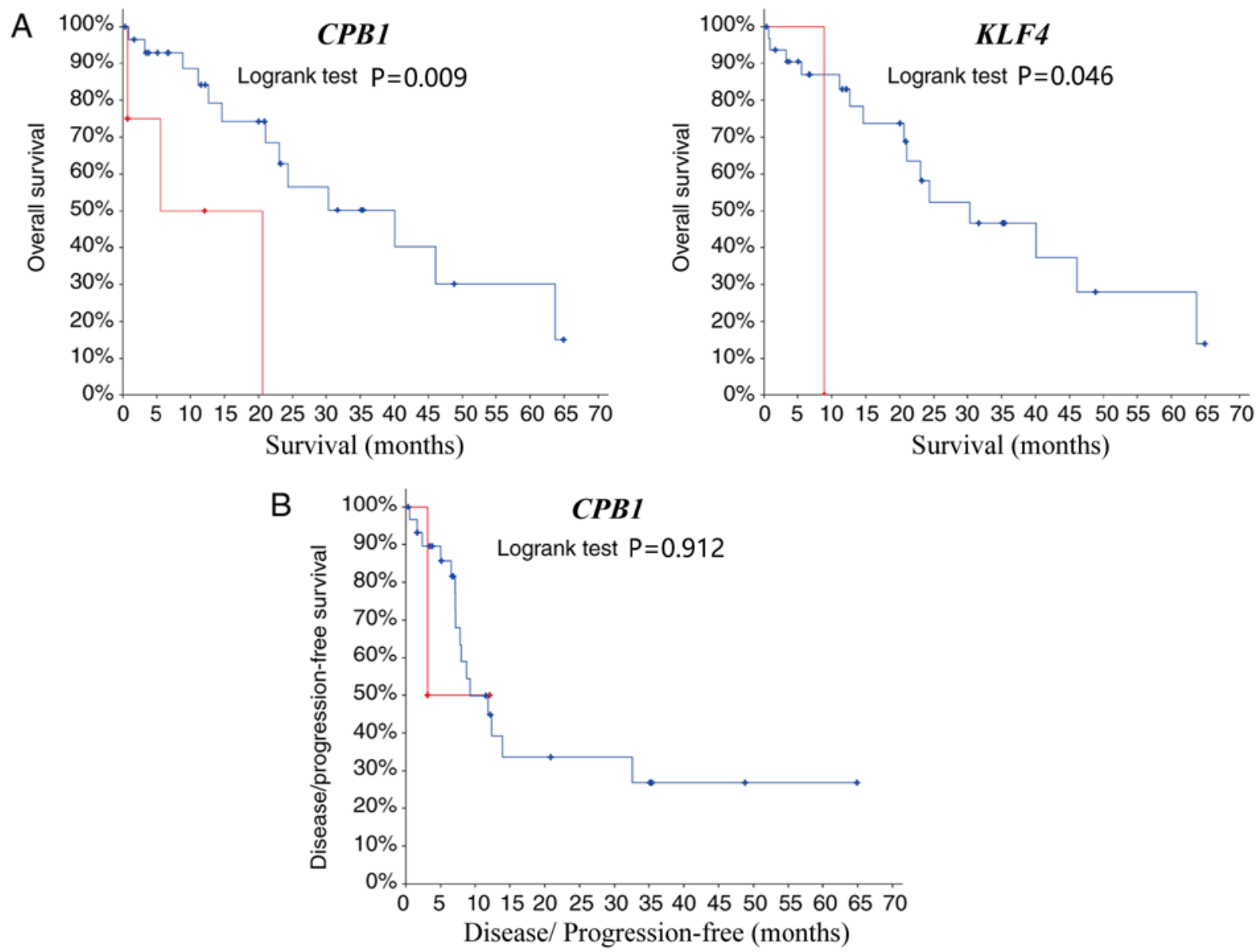

Figure 5. (A) Overall and (B) disease-free survival time analyses of $C P B 1$ and $K L F 4$ were performed using the cBioPortal online platform. P $<0.05$ was considered to indicate a statistically significant difference. $C P B 1$, carboxypeptidase B1; KLF4, Kruppel like factor 4.

have a significant effect on patient outcome are presented in Fig. S1. Patients with CCA with carboxypeptidase B1 (CPBI) and KLF4 (Kruppel like factor 4) upregulation, had lower overall survival rates compared with patients without alterations ( $\mathrm{P}=0.008$ and 0.046 respectively; Fig. 5A). However, whilst $C P B 1$ upregulation was significantly associated with lower overall survival, it was not associated with disease-free survival ( $\mathrm{P}=0.912$; Fig. 5B). The Kaplan-Meier estimate could not be used for the disease-free survival analysis of KLF4 due to the lack of clinical data on the 'disease-free survival time' of patients with the upregulation.

\section{Discussion}

CCA is the second most common hepatobiliary malignancy after hepatocellular carcinoma (24). The main etiological factors of CCA include primary sclerosing cholangitis, cirrhosis and hepatitis C and B infections (25). While chronic infection and inflammation in the bile ducts play a major role in CCA, the molecular mechanisms involved in CCA remain poorly understood. The most commonly mutated genes in CCA are SMAD4, TP53, KRAS, BAPl, isocitrate dehydrogenase $[\mathrm{NADP}(+)] 1$ cytosolic, isocitrate dehydrogenase [NADP(+)] 2 mitochondrial and roundabout guidance receptor
$2(2,4)$. In addition, CCA has been reported to be associated with inflammation, the growth factor signaling pathway, cell signaling pathways and epigenetic regulation $(3,26)$. There are currently no effective clinical biomarkers and targeted molecular therapies for the early diagnosis and treatment of CCA; consequently, the 5 -year survival rate is $\sim 10 \%$ (27). There is therefore a requirement of the identification of diagnostic markers for CCA. Microarray technology has previously been used to identify novel biomarkers in various diseases and may also be applied to uncover diagnostic markers in CCA (10).

In the present study, two microarray datasets (GSE26566 and GSE89749) were obtained from the GEO and analyzed to identify DEGs between CCA and normal bile duct samples. In total, 159 DEGs (135 upregulated and 24 downregulated) were identified. GO and KEGG enrichment analyses were performed to investigate interactions among the DEGs and a PPI network was constructed, revealing 10 hub genes. The PPI network demonstrated that interleukin-6 (IL-6) had the largest number of nodes (20 nodes) and directly interacted with c-Myc, somatostatin, C-X-C motif chemokine 12 (CXCL12), neuropeptide Y (NPY) and LPAR1, suggesting that IL-6 may serve an important role in CCA. Consistent with the results obtained in the current study, a recent study demonstrated that increased IL- 6 expression plays a central 
role in the pathogenesis and progression of CCA (28). In addition, the circulating level of IL-6 in patients with CCA was reported to be increased compared with healthy controls (29). Moreover, overexpression of IL-6 promotes cell survival in malignant cholangiocytes and enhances tumor growth (30). A previous study reported that a combination of increased levels of leucine-rich $\alpha$-2-glycoprotein 1 , carbohydrate antigen 19-9 and IL-6 in the serum could be sued to discriminate between biliary tract cancer (CCA and gallbladder carcinoma) and benign biliary disease (31). $c-M Y C$ is a proto-oncogene and encodes a nuclear phosphoprotein, which primarily regulates apoptosis, cell cycle progression and cellular transformation (32). A previous study showed that $c-M Y C$ is upregulated in human CCA (33). Furthermore, cyclin D1, a c-Myc target gene, is a molecular biomarker of CCA (34). Knockdown of $c-M y c$ significantly reduced the extent of cholangiofibrosis and cholangioma in vivo, highlighting the importance of $c-M y c$ in the progression of CCA (35). C-X-C motif chemokine receptor 4 (CXCR4) selectively binds CXCL12 and the CXCR4/CXCL12 axis has been shown to be involved in tumorigenesis, cell proliferation and angiogenesis in CCA (36). A recent study suggested that serum CXCL12 levels may serve as a potential biomarker for predicting the clinical outcome in CCA (37). However, in the present study, elevated CXCL12 levels were not significantly associated with disease-free or overall survival. This may be due to the cBioPortal survival analyses performed, which were based on the association between gene mutation and prognosis, whereas high expression in serum is generally caused by a mutation or upregulation (38).

The association between CCA and the hub genes $N P Y$, $L P A R 1$, phospholipase A2 group IB, $C P B 1$, pancreatic lipase and $K L F 4$ has not been widely reported. $N P Y$ expression has been shown to be upregulated in CCA $(39,40)$, therefore regulating $N P Y$ expression may be beneficial for the treatment of CCA. Previous research demonstrated that KLF4 and microRNA-21 play a key role in mediating the EMT in CCA cells via the Akt/extracellular signal-regulated protein kinase 1 and 2 signaling pathway (41). Hierarchical clustering revealed that the hub genes identified in the present study may be used to differentiate CCA from normal bile duct samples. Furthermore, upregulation of $C P B 1$ and KLF4 was associated with a lower overall survival rate, suggesting that the aforementioned genes may serve important roles in the progression of CCA.

In summary, the present study identified DEGs that may be involved in the carcinogenesis or progression of CCA. A total of 159 DEGs and 10 hub genes were identified, which following further investigation may serve as diagnostic biomarkers and novel therapeutic targets for CCA.

\section{Acknowledgements}

Not applicable.

\section{Funding}

The present study was financially supported by the Natural Science Foundation of Ningbo City (grant no. 2017A610259), the Huamei Research Foundation (grant no. 2017HMKY07) and the Medicine and Health Sciences Research Foundation of Zhejiang Province (grant no. 2019KY177). Additionally, the study was sponsored by the K.C. Wong Magna Fund of Ningbo University.

\section{Availability of data and materials}

The datasets used and/or analyzed during the present study are available from Gene Expression Omnibus; ncbi.nlm.nih. gov/geo/query/acc.cgi?acc=GSE26566 and ncbi.nlm.nih.gov/ geo/query/acc.cgi?acc=GSE89749.

\section{Authors' contributions}

JT designed the study. RH acquired and interpreted the datasets. MZ performed data analysis. XM and YZ performed the survival analysis. All authors read and approved the final manuscript.

\section{Ethics approval and consent to participate}

Not applicable.

\section{Patient consent for publication}

Not applicable.

\section{Competing interests}

The authors declare that they have no competing interests.

\section{References}

1. Saha SK, Zhu AX, Fuchs CS and Brooks GA: Forty-Year trends in cholangiocarcinoma incidence in the U.S.: Intrahepatic disease on the rise. Oncologist 21: 594-599, 2016.

2. Kongpetch S, Jusakul A, Ong CK, Lim WK, Rozen SG, Tan P and The BT: Pathogenesis of cholangiocarcinoma: From genetics to signalling pathways. Best Pract Res Clin Gastroenterol 29: 233-244, 2015.

3. Razumilava N and Gores GJ: Cholangiocarcinoma. Lancet 383: 2168-2179, 2014.

4. Valle JW, Lamarca A, Goyal L, Barriuso J and Zhu AX: New horizons for precision medicine in biliary tract cancers. Cancer Discov 7: 943-962, 2017.

5. Ikeno Y, Seo S, Iwaisako K, Yoh T, Nakamoto Y, Fuji H, Taura K, Okajima H, Kaido T, Sakaguchi S and Uemoto S: Preoperative metabolic tumor volume of intrahepatic cholangiocarcinoma measured by ${ }^{18} \mathrm{~F}-\mathrm{FDG}$-PET is associated with the KRAS mutation status and prognosis. J Transl Med 16: 95, 2018.

6. Hill MA, Alexander WB, Guo B, Kato Y, Patra K, O'Dell MR, McCall MN, Whitney-Miller CL, Bardeesy N and Hezel AF: Kras and Tp53 Mutations cause cholangiocyte- and hepatocyte-derived cholangiocarcinoma. Cancer Res 78: 4445-4451, 2018.

7. Mahipal A, Kommalapati A, Tella SH, Lim A and Kim R: Novel targeted treatment options for advanced cholangiocarcinoma. Expert Opin Invest Drugs 27: 709-720, 2018.

8. Qiao P, Li G, Bi W, Yang L, Yao L and Wu D: microRNA-34a inhibits epithelial mesenchymal transition in human cholangiocarcinoma by targeting Smad4 through transforming growth factor-beta/Smad pathway. BMC Cancer 15: 469, 2015.

9. Chan-On W, Nairismagi ML, Ong CK, Lim WK, Dima S, Pairojkul C, Lim KH, McPherson JR, Cutcutache I, Heng HL, et al: Exome sequencing identifies distinct mutational patterns in liver fluke-related and non-infection-related bile duct cancers. Nat Genet 45: 1474-1478, 2013.

10. Vogelstein B, Papadopoulos N, Velculescu VE, Zhou S, Diaz LA $\mathrm{Jr}$ and Kinzler KW: Cancer genome landscapes. Science 339: 1546-1558, 2013. 
11. Andersen JB, Spee B, Blechacz BR, Avital I, Komuta M, Barbour A, Conner EA, Gillen MC, Roskams T, Roberts LR, et al: Genomic and genetic characterization of cholangiocarcinoma identifies therapeutic targets for tyrosine kinase inhibitors. Gastroenterology 142: 1021-1031.e15, 2012.

12. Jusakul A, Cutcutache I, Yong CH, Lim JQ, Huang MN, Padmanabhan N, Nellore V, Kongpetch S, Ng AWT, Ng LM, et al: Whole-Genome and epigenomic landscapes of etiologically distinct subtypes of cholangiocarcinoma. Cancer Discov 7 : $1116-1135,2017$.

13. Zou S, Li J, Zhou H, Frech C, Jiang X, Chu JS, Zhao X, Li Y, Li Q, Wang H, et al: Mutational landscape of intrahepatic cholangiocarcinoma. Nat Commun 5: 5696, 2014.

14. Guo YC, Bao YH, Ma M and Yang WC: Identification of key candidate genes and pathways in colorectal cancer by integrated bioinformatical analysis. Int J Mol Sci 18: Pii E722, 2017.

15. Fu Q, Yang F, Zhao J, Yang X, Xiang T, Huai G, Zhang J, Wei L, Deng S and Yang H: Bioinformatical identification of key pathways and genes in human hepatocellular carcinoma after CSN5 depletion. Cell Signal 49: 79-86, 2018.

16. Huang DW, Sherman BT, Tan Q, Collins JR, Alvord WG, Roayaei J, Stephens R, Baseler MW, Lane HC and Lempicki RA: The DAVID gene functional classification tool: A novel biological module-centric algorithm to functionally analyze large gene lists. Genome Biol 8: R183, 2007.

17. Smoot ME, Ono K, Ruscheinski J, Wang PL and Ideker T: Cytoscape 2.8: New features for data integration and network visualization. Bioinformatics 27: 431-432, 2011.

18. Szklarczyk D, Morris JH, Cook H, Kuhn M, Wyder S, Simonovic M, Santos A, Doncheva NT, Roth A, Bork P, et al: The STRING database in 2017: Quality-controlled protein-protein association networks, made broadly accessible. Nucleic Acids Res 45 (D1): D362-D368, 2017.

19. Bader GD and Hogue CW: An automated method for finding molecular complexes in large protein interaction networks. BMC Bioinformatics 4: 2, 2003.

20. Tripathi S, Pohl MO, Zhou Y, Rodriguez-Frandsen A, Wang G, Stein DA, Moulton HM, DeJesus P, Che J, Mulder LC, et al: Meta- and orthogonal integration of influenza "OMICs" data defines a role for UBR4 in Virus budding. Cell Host Microbe 18 723-735, 2015

21. Cerami E, Gao J, Dogrusoz U, Gross BE, Sumer SO, Aksoy BA, Jacobsen A, Byrne CJ, Heuer ML, Larsson E, et al: The cBio cancer genomics portal: An open platform for exploring multidimensional cancer genomics data. Cancer Discov 2: 401-404, 2012

22. Gao J, Aksoy BA, Dogrusoz U, Dresdner G, Gross B, Sumer SO, Sun Y, Jacobsen A, Sinha R, Larsson E, et al: Integrative analysis of complex cancer genomics and clinical profiles using the cBioPortal. Sci Signal 6: pl1, 2013.

23. Zhang K, Yu J, Yu XL, Han ZY, Cheng ZG, Liu FY and Liang P: Clinical and survival outcomes of percutaneous microwave ablation for intrahepatic cholangiocarcinoma. Int J Hyperthermia 34: 292-297, 2018

24. Rizvi S, Khan SA, Hallemeier CL, Kelley RK and Gores GJ Cholangiocarcinoma - evolving concepts and therapeutic strategies. Nat Rev Clin Oncol 15: 95-111, 2018.

25. Bagante F, Gamblin TC and Pawlik TM: Cholangiocarcinoma risk factors and the potential role of aspirin. Hepatology 64 708-710, 2016

26. Rizvi S and Gores GJ: Pathogenesis, diagnosis, and management of cholangiocarcinoma. Gastroenterology 145: 1215-1229, 2013.

27. Nakamura H, Arai Y, Totoki Y, Shirota T, Elzawahry A, Kato M, Hama N, Hosoda F, Urushidate T, Ohashi S, et al: Genomic spectra of biliary tract cancer. Nat Genet 47: 1003-1010, 2015.
28. Tanjak P, Thiantanawat A, Watcharasit $\mathrm{P}$ and Satayavivad J: Genistein reduces the activation of AKT and EGFR, and the production of IL6 in cholangiocarcinoma cells involving estrogen and estrogen receptors. Int J Oncol 53: 177-188, 2018.

29. Cheon YK, Cho YD, Moon JH, Jang JY, Kim YS, Kim YS Lee MS, Lee JS and Shim CS: Diagnostic utility of interleukin-6 (IL-6) for primary bile duct cancer and changes in serum IL-6 levels following photodynamic therapy. Am J Gastroenterol 102: 2164-2170, 2007.

30. Meng F, Yamagiwa Y, Ueno Y and Patel T: Over-expression of interleukin-6 enhances cell survival and transformed cell growth in human malignant cholangiocytes. J Hepatol 44: 1055-1065, 2006.

31. Sandanayake NS, Sinclair J, Andreola F, Chapman MH, Xue A, Webster GJ, Clarkson A, Gill A, Norton ID, Smith RC, et al: A combination of serum leucine-rich alpha-2-glycoprotein 1, CA19-9 and interleukin-6 differentiate biliary tract cancer from benign biliary strictures. Br J Cancer 105: 1370-1378, 2011.

32. Stine ZE, Walton ZE, Altman BJ, Hsieh AL and Dang CV: MYC, metabolism, and cancer. Cancer Discov 5: 1024-1039, 2015.

33. Boulter L, Guest RV, Kendall TJ, Wilson DH, Wojtacha D, Robson AJ, Ridgway RA, Samuel K, Van Rooijen N, Barry ST, et al: WNT signaling drives cholangiocarcinoma growth and can be pharmacologically inhibited. J Clin Invest 125: 1269-1285, 2015.

34. Yang H, Li TW, Peng J, Tang X, Ko KS, Xia M and Aller MA: A mouse model of cholestasis-associated cholangiocarcinoma and transcription factors involved in progression. Gastroenterology 141: 378-388, 388.e1-e4, 2011.

35. Yang H, Liu T, Wang J, Li TW, Fan W, Peng H, Krishnan A, Gores GJ, Mato JM and Lu SC: Deregulated methionine adenosyltransferase $\alpha 1, \mathrm{c}-\mathrm{Myc}$, and Maf proteins together promote cholangiocarcinoma growth in mice and humans (double dagger). Hepatology 64: 439-455, 2016.

36. Zhao S, Wang J and Qin C: Blockade of CXCL12/CXCR4 signaling inhibits intrahepatic cholangiocarcinoma progression and metastasis via inactivation of canonical Wnt pathway. J Exp Clin Cancer Res 33: 103, 2014.

37. Lee SJ, Kim JE, Kim ST, Lee J, Park SH, Park JO, Kang WK, Park YS and Lim HY: The correlation between serum Chemokines and clinical outcome in patients with advanced biliary tract cancer. Transl Oncol 11: 353-357, 2018.

38. Li L, Lei QS, Zhang SJ, Kong LN and Qin B: Screening and identification of key biomarkers in hepatocellular carcinoma: Evidence from bioinformatic analysis. Oncol Rep 38: 2607-2618, 2017.

39. DeMorrow S, Onori P, Venter J, Invernizzi P, Frampton G, White M, Franchitto A, Kopriva S, Bernuzzi F, Francis H, et al: Neuropeptide Y inhibits cholangiocarcinoma cell growth and invasion. Am J Physiol Cell Physiol 300: C1078-C1089, 2011.

40. DeMorrow S, Onori P, Venter J, Leyva-Illades D, Francis H, Frampton G, Pae HY, Quinn M, Onori P, Glaser S, et al: Neuropeptide Y inhibits biliary hyperplasia of cholestatic rats by paracrine and autocrine mechanisms. Am J Physiol Gastrointest Liver Physiol 305: G250-G257, 2013.

41. Liu CH, Huang Q, Jin ZY, Zhu CL, Liu Z and Wang C: miR-21 and KLF4 jointly augment epithelial-mesenchymal transition via the Akt/ERK1/2 pathway. Int J Oncol 50: 1109-1115, 2017.

(7) $\Theta$ This work is licensed under a Creative Commons Attribution-NonCommercial-NoDerivatives 4.0 International (CC BY-NC-ND 4.0) License. 